\title{
LOS MOTIVOS DE UNA FUGAZ RESURRECCIÓN: EL MÉTODO HISTÓRICO NATURAL EN LA OBRA DE K.W. STARK (1787-1845) Y F. JAHN (1804-1859)
}

\author{
Luis Montiel
}

(Universidad Complutense)

\section{RESUMEN}

En el presente trabajo se analizan las propuestas metodológicas de dos médicos alemanes de la primera mitad del siglo XIX, tradicionalmente vinculados a la llamada "Escuela historiconatural". Se intenta, especialmente, evaluar la importancia concedida por cada uno de ellos al "método historiconatural" aplicado a la clasificación de las enfermedades, tomando en consideración que ambos defienden una concépción ontologista o "parasitaria" de las mismas. El estudio muestra que este empeño responde al desafío, planteado por los seguidores de la Naturphilosophie de Schelling, de convertir a la medicina en una ciencia natural, así como que este empeño estaba abocado al fracaso a causa de la contradicción existente entre la utilización del método clasificatorio, propio de la Historia Natural dieciochesca, y la necesidad de una fundamentación fisiológica de la nosología, más acorde con la naciente Biología, que disminuirá grandemente la solidez epistemológica del concepto de especie morbosa. Este mismo desequilibrio se pondrá de manifiesto, en el aspecto más propiamente médico, en las dudas a la hora de conceder prioridad epistemológica a la físiología o a la clínica.

\begin{abstract}
This Work analyses the methodological proposals of two German doctors in the first half of the XIX century, traditionally linked to the so-called "Naturalistic School". It aims especially to evaluate the importance given by each to the "Naturalistic method" applied to the classification of these diseases, taking into account the fact that both defended an ontologistic or "parasitic" conception of the same. This study illustrates how this undertaking responds to the challenge, expounded by the followers of Schelling's Naturphilosophie, of converting medicine into a natural science. Likewise, this undertaking was doomed to failure due to the existing contradiction between the utilization to the classificatory method, characteristic of eighteenth.century Natural History, and the need for a physiological foundation of nosology, more in acoordance with the nascent Biology, which will greatly reduce the epistemological soundness of the concept of morbid species. This very imbalance will be revealed, in the strictly medical aspect, in the doubts when giving epistemological priority to physiology or to the clinical observation.
\end{abstract}




\section{LA NATURHISTORISCHE SCHULE EN LA HISTORIOGRAFÍA MÉDICA.}

La llamada "escuela historiconatural" — naturhistorische Schule - no es bien conocida entre nosotros. Pocos historiadores de la medicina españoles se han ocupado de ella. Por su parte, la historiografía médica extranjera tampoco le ha dedicado una atención exhaustiva. Este menguado interés resulta comprensible teniendo en cuenta que, excepción hecha de Schönlein, los miembros de la sedicente "escuela historiconatural" no desempeñaron un papel relevante en el devenir de la medicina como ciencia. Sin embargo, como explicaré en breve, la mera existencia de este movimiento plantea problemas muy excitantes al historiador de la medicina. Antes de llegar a ello, intentaré dar una perspectiva de la situación de la investigación en este campo.

Vengamos a la bibliografía en castellano: de la naturhistorische Schule se ocupan, en primer término, las obras enciclopédicas de Laín Entralgo. Dada la época en que fueron escritas - La historia clínica en 1950, $2^{\mathrm{a}}$ ed. 1961 y la Historia de la medicina moderna y contemporánea en $1954,2^{\mathrm{a}}$ ed. 1963-, así como el ya citado carácter enciclopédico de ambas obras, la información al respecto es escasa y derivada de la que podía suministrar la historiografía alemana anterior a esos años. El extenso y documentado trabajo de López Piñero sobre los sistemas nosológicos publicado en Asclepio en $1961^{1}$ alcanza mayor precisión en algunos puntos, aunque la atención prestada a los autores de la naturhistorische Schule es forzosamente limitada y se apoya, igualmente, en la bibliografía disponible en ese momento que, por ejemplo, para el caso de Schönlein, contempla solamente los escritos apócrifos ${ }^{2}$.

En lo anterior no debe verse menosprecio alguno, y aún menos afán de resaltar la propia originalidad sobre el fondo de la crítica a los maestros ${ }^{3}$. No hay que olvidar que ni tan siquiera en la época de su florecimiento la escuela ostentaba unos rasgos perfectamente definidos, lo que ha complicado bastante la tarea de los mismos historiadores alemanes. El grupo de médicos que se da a sí mismo, o acepta por bueno, el nombre de "escuela historiconatural" dista de ser homogéneo, de manera que es aquí, 93.

1 LOPEZ PIÑERo, J.M. (1961). "Los sistemas nosológicos del siglo XVIII". Asclepio, XIII, pp. 65 -

2 Las dos obras citadas en la bibliografía (la edición de 1839 de la Allgemeine und spezielle Pathologie und Therapie y los Klinische Vorträge in dem Charité Krankenhaus zu Berlin) fueron redactadas y publicadas por algunos de sus oyentes. La Patología, en concreto, fue desautorizada por el propio Schönlein, aunque cabe preguntarse si el motivo fundamental fue el económico.

3 Conviene, a este respecto, señalar que un libro mucho más reciente, dedicado, además, exclusivamente a la medicina clínica, consigue introducir una suma de errores groseros y simplificaciones intolerables muy superior en cantidad y calidad a cuanto pueda encontrarse en toda la bibliografía precedente, iy solamente en trece líneas!. Cfr. Altschule, M. (1989). Essays on the Rise and Decline of Bedside Medicine. Philadelphia-London, p. 290. 
precisamente, donde radica el primer problema historiográfico, que desde luego no ha escapado a los más sutiles analistas de este movimiento: en saber si puede hablarse con propiedad de una "escuela", por existir unos supuestos fundamentales compartidos por todos los autores a los que se considera - 0 que se consideran a sí mismos- miembros de este grupo. Un trabajo, bastante reciente, de J. Bleker ${ }^{4}$, suministra las respuestas más atinadas a esta pregunta, de modo que, en mi opinión, constituye el punto de partida de cualquier investigación actual sobre la Naturhistorische Schule.

Frecuentemente se ha atribuído a los miembros de esta escuela el sostenimiento, en lo conceptual, de una teoría ontologista acerca de la enfermedad, así como, en lo metodológico, el retorno a una medicina basada en la observación y la clasificación. Hay que decir que ni Laín ni López Piñero han sostenido este punto de vista, introduciendo en su apreciación matices más sutiles y realistas. J. Bleker considera, en síntesis, que la Parasitenlehre - la doctrina que considera toda enfermedad como un parásito, es decir, una modalidad del ontologismo nosológico-, no fue aceptada por la totalidad de los médicos que se adscriben a este movimiento, en particular por J.L. Schönlein, a quien sus propios contemporáneos consideran el fundador de la escuela ${ }^{5}$. Por el contrario, el método historiconatural, basado en la observación y en la clasificación, parece haber sido, de hecho, compartido por todos, ontologistas y no ontologistas, aunque, a juicio de esta autora, tampoco debe considerarse como el supuesto fundamental de la "Escuela historiconatural"6, pese a lo que podría indicar su nombre. El trabajo de Bleker pretende, ante todo, poner de relieve las diversidades internas de esta presunta "escuela", así como - tal como indica el subtítulo de su libro- subrayar las aportaciones de alguno de sus miembros, muy en primer término Schönlein, a la medicina clínica alemana y mundial.

Mi pretensión, desde esta perspectiva, no es otra que la de comprender el papel epistemológico concedido a las clasificaciones morbosas por unos médicos que, en gran medida - lo adelanto-, son herederos directos de aquellos que negaron la utilidad de las mismas para hacer de la medicina una ciencia. Para ello debo volver a lo que fue hasta hace poco el punto de partida para un historiador de la medicina

4 BLEKER, J. (1981). Die naturhistorische Schule 1825-1845. Ein Beitrag zur Geschichte der klinischen Medizin in Deutschland. Stuttgart \& New York. Veinticuatro horas antes de celebrarse las jornadas a las que presenté este trabajo tuve noticia, a través de una recensión, de la existencia de un libro cuya tercera parte estudia el papel de la Naturhistorische Schule - al parecer sensu stricto (Schönlein, Fuchs, Cannstat y Siebert)—en el desarrollo del método clínico en Alemania. MAEHLE, A.H. (1995). "Volker Hess. Von der semiotischen zur diagnostischen Medizin: Die Entstehung der klinischen Methode zwischen 1750 und 1850. Abhandlungen zur Geschichte der Medizin und der Naturwissenschaften, 66. Husum, 1993". Medical History, 39 (4), pp. 513-514.

5 BLEKER, J. (1981), pp. 132-133.

6 Ibid. 


\section{LUIS MONTIEL}

español: la idea de que muchos de estos autores, en especial Schönlein, propiciaron, en tanto que "generación intermedia", el tránsito de la Naturphilosophie a la $\mathrm{Na}$ turwissenschaft en la medicina. La propia idea de "generación intermedia" orienta, aunque involuntariamente, al investigador en un sentido supuestamente "progresivo", que hace pensar más en el destino que en la procedencia. Y, a mi juicio, no puede comprenderse la mera existencia de una "escuela historiconatural" - tan confusa como se quiera - si se pierde de vista la consideración retrospectiva ${ }^{7}$.

\section{INTEMPESTIVIDAD DE LAS NOSOLOGÍAS.}

Como acabo de adelantar, los médicos de cuya obra voy a ocuparme suceden a una generación que asumió como tarea fundamental la conversión de la medicina en ciencia, rechazando, para ello, opciones propias de la medicina del siglo XVIII. Como signo de ese pasado reciente pueden verse las siguientes líneas, en las que un médico del período romántico declara su talante rupturista:

\footnotetext{
Hasta ahora no existe un sistema de la Medicina que responda con propiedad a este nombre, es decir, una obra en la cual las leyes generales de la vida sean deducidas de los principios fundamentales de la misma, comprobadas en la enfermedad y en sus manifestaciones individuales, y mediante el cual puedan ser ordenadas y estudiadas las enfermedades individuales. Los escritos que, en tiempos anteriores, aparecieron bajo este título eran recopilaciones de lo semejante sin un principio internos.
}

Con esta declaración, el médico D. G. Kieser (1779-1862) —a quien, en este como en otros aspectos, cabe considerar como portavoz del amplio movimiento de renovación promovido por la Naturphilosophie de Schelling ${ }^{9}$ - se despega de la

7 A este respecto hay que señalar el acierto de uno de los pocos estudios clásicos sobre la nosología historiconatural -el de W. Karst-, por más que, en la actualidad, haya sido superado por el citado de Bleker. El capítulo consagrado a la "escuela historiconatural" comienza subrayando resueltamente la continuidad existente entre ésta y la precedente medicina de la Naturphilosophie. Cfr. KARST, W. (1941). Zur Geschichte der "Natürlichen Krankheitssysteme". Abhandlungen zur Geschichte der Medizin und der Naturwissenschaften, 37. Berlin. Cito por el reprint de 1979, Nendel/Liechtenstein, p. 45.

8 KIESER, D.G. (1817). System der Medizin zum Gebrauche bei akademischen Vorlesungen und fiir practische Ärzte, Bd. I. Halle, p. III

9 RothSCHUH, K.E. (1978). "Dietrich Georg Kiesers System der Medizin (1817/19). En: Konzepte der Medizin in Vergangenheit und Gegenwart. Stuttgart, pp. 409-416. Cfr. BREDNOW, W. (1970) Dietrich Georg Kieser. Sein Leben und Werk. Wiesbaden; BREDNOW, W. (1971). "Wandlungen der Wissenschaftslehre im Leben des Jenaer Professors D.G. Kieser". En: Das Humanum und die Wissenschaft, pp. 239-258. 
medicina clasificadora del siglo XVIII. La medicina filosófica ha lanzado su interdicto sobre las nosologías ${ }^{10}$, y eso, en Alemania, es algo muy serio, incluso para aquellos que, pocos años más tarde, abominarán de la medicina de orientación filosoficonatural por juzgarla altamente especulativa. Así, C.A. Wunderlich, considerado como uno de los fundadores de la medicina de orientación cientificonatural, de laboratorio, se mostrará, todavía, más agradecido al estímulo intelectual representado por la obra de Schelling que a los nosógrafos clasificadores del pasado siglo o a los presuntos empiristas con los que convive" ${ }^{11}$. Y no es un caso aislado: el rechazo de la medicina filosófica no es tan general como podría creerse, de lo que es buena prueba el siguiente dato. Cuando, en una lección inaugural publicada en 1838, el médico C.F. von Marcus se ocupe "del actual nivel de la medicina", sostendrá que la base filosófica, y en especial la suministrada por la Naturphilosophie, ha ennoblecido a la medicina, pues ha hecho salir al médico del empirismo más tosco en busca de las demás ciencias naturales ${ }^{12}$. A la vista de lo anterior, el anatema de Kieser no parece baladí.

Cierto es que los tratadistas de Patología, incluídos los Naturphilosophen, no prescindirán de la nosotaxia, pero sin concederle, en general, preeminencia epistemológica alguna. Sin embargo, tal como hemos advertido desde el comienzo, precisamente en el período inmediatamente ulterior a aquél en que florece la Naturphilosophie, algunos autores se agruparán en torno al criterio taxonómico para dar su personal respuesta a la crisis de fundamentos de la medicina en tanto que ciencia que la filosofía natural de Schelling pretendía, así mismo, remediar ${ }^{13}$. Cabe, entonces, suponer que aquellos médicos alemanes que se aventuren a reclamar alguna validez científica para el método nosográfico deberán hacerlo desde supuestos diferentes a los de épocas pasadas, e incluso a los de sus contemporáneos no alemanes. Dada la diversidad interna de la escuela, he decidido centrar mi atención en K.W. Stark

11) El término "nosología", tal como aquí se utiliza, requiere alguna aclaración: si bien cl diccionario médico de Dechambre advierte, de manera explícita, que este vocablo no implica en modo alguno la idea de clasificación - lo que correspondería más bien al término "nosografía"- (vol. 13, p. 356), en la práctica la obra de Pinel realiza este vínculo que, en adelante, opera en la mente de quien lo utiliza, de modo que es oportuno utilizarlo en este sentido al referirse a la época objeto del presente estudio, como advierte BLEKER, J. (1981), p. 9.

11 Temkin, O. (1966). "Wunderlich, Schelling and the History of Medicine". Gesnerus, 23, pp. 188195. Publicado ulteriormente (1977) en The Double Face of Janus and Other Essays in the History of Medicine. Baltimore and London, pp. 246-251.

12 MARCuS, C.F.V. (1838). Über die Entwicklung und der gegenwärtigen Stadpunct der Medicin. Eine einleitende Vorlesung zur medicinischen Klinik. Würzburg, pp. 9-11.

13 Véase al respecto el capítulo IV, "Los médicos alemanes ante la crisis de fundamentos de la medicina" de: ARQUIOLA, E.; MONTIEL, L. (1993). La corona de las ciencias naturales. La medicina en el tránsito del siglo XVIII al XIX. Madrid, CSIC, pp. 77-89, así como la bibliografía en él citada. 


\section{LUIS MONTIEL}

(1787-1845) y F. Jahn (1804-1859)14, quienes comparten como postulado el ontologismo nosológico, y a quienes la citada historiadora alemana, rompiendo con la tradición, aparta de la naturhistorische Schule propiamente dicha ${ }^{15}$.

\section{El MÉTOdo CLASIFICATORIO EN EL PENSAMIENTO DE K.W. STARK.}

La consideración retrospectiva que propongo es especialmente inexcusable en el caso de Stark, el auténtico fundador de la Parasitenlehre. La indudable influencia de ese mismo Kieser, cuya descalificación de las nosologías hemos visto páginas atrás, resulta evidente para quien conozca la obra del Naturphilosoph ${ }^{16}$. No en vano es Kieser quien acuña los términos Afterorganisation - seudoorganización- y Afterorganismus - seudoorganismo- para caracterizar genéricamente al proceso morboso ${ }^{17}$, de modo que la tesis central de la doctrina de Stark parece derivar naturalmente del pensamiento del colega aún vivo y activo. En mi opinión, la consideración de la enfermedad como organismo no equivale a una pura identidad en el caso de Kieser, que no es ontologista en el sentido fuerte del término, dado que los conceptos por él acuñados y asumidos luego por Stark y Jahn tienen, en su obra, un valor fundamentalmente orientativo, al que me atrevería a llamar metafórico; o más bien, forzando un poco la mano - lo que creo permisible por lo que he encontrado en el propio Kieser y en otros Naturphilosophen-, podría decirse que tiene un valor "regulativo", en el sentido kantiano, y no "constitutivo", como termina ocurriendo en los parasitistas propiamente dichos. Sea ello como fuere, en el caso de Stark la defensa de las clasificaciones tendrá, a causa de esa filiación que acabo de señalar, que reposar sobre un fundamento filosófico aceptable, es decir, susceptible de ser legitimado por una filosofía natural poco o nada discrepante de la profesada por Kieser ${ }^{18}$. A ello dedica la tercera sección del segundo tomo de su Allgemeine Pathologie —Patología

\footnotetext{
14 También estos autores, especialmente Stark, son estudiados en el libro de Bleker. Llegado el caso pondré de relieve lo que, en mi exposición, proceda de esta autora. Sobre Jahn existe un antiguo trabajo, muy útil para obtener una visión sumaria de su pensamiento, así como para recabar algunos datos de su biografía: LeHMANN, H. (1936). Die Krankheits- und Heilungslehre des meiningischen Hofmedicus Ferdinand Jahn. Ein Beitrag zur Kenntnis der naturhistorischen Schule in der Medizin. Abhandlungen zur Geschichte der Medizin und der Naturwissenschaften, 14. Berlin, 1936.

15 Ibid, p. 132.

16 También ha sido puesta de relieve por BLEKER, J. (1981), pp. 33-36.

17 KIESER, D.G. (1817), Bd. I, pp. 112-113.

18 Otro historiador alemán, A. Bauer, ha puesto de relieve la influencia ejercida sobre Stark por el uso, sumamente prestigiado, del método analógico en el estudio de la anatomía. BAUER, A. (1985). "Die Krankheitslehre von Karl Wilhelm Stark (1787-1845): ontologische Pathologie als Analogiemodell". Sudhoffs Archiv, 69 (2), pp. 129-153.
} 
general, 1838-, obra de elocuente subtítulo: allgemeine Naturlehre der Krankheit -Doctrina natural de la enfermedad - La reivindicación que allí hace se apoya fundamentalmente en la utilidad instrumental de las clasificaciones, que permiten aislar lo singular e identificarlo, y que facilitan la comunicación del saber patológico entre los médicos, aun cuando, para que este saber sea científico, las singularidades descritas - advierte - deben serlo desde una perspectiva que dé cuenta del fondo común compartido por los diferentes individuos. Sólo mediante la identificación de esa comunidad de base adquiere sentido la clasificación. En esto, Stark manifiesta haber entendido el mensaje de la medicina filosófica de sus predecesores ${ }^{19}$, poniéndose, por tanto, al resguardo de críticas como la de Kieser.

Con todo, la tarea clasificatoria presenta, para el caso de las enfermedades, una dificultad añadida a las que se reconocen a las taxonomías botánica y zoológica, que se desprende de la concepción de la enfermedad como parásito:

El individuo-enfermedad existe solamente como parásito y frecuentemente está tan íntima, dinámica y materialmente unido a su huésped, que la distinción entre ambos implica grandes dificultades ${ }^{20}$.

Como muy gráficamente explica poco más lejos, a ningún botánico digno de ese nombre se le ocurriría clasificar como un solo ser al árbol y a la planta parásita que de él se nutre, por más que las interacciones nacidas de su convivencia, los cambios funcionales y estructurales que el parásito produce en el huésped, puedan dar la impresión de que nos encontramos ante una tercera - y diferente - forma de vida. Precisamente esta distinción arrastra consecuencias muy notables en relación con el criterio clasificatorio, pues se coloca por encima de nociones hasta entonces fundamentales - la enfermedad como passio (Galeno) o como reactio (conamen, Sydenham) — que hacían al síntoma objeto de una consideración opuesta, pero siempre elevada, mientras que, para Stark, y a la vista de lo señalado, el síntoma, ya sea "activo" o "pasivo", carece de valor en tanto que resultado de la contienda de dos

19 Cfr. lo que al respecto habían sostenido, por ejemplo, Reil y Kieser. El primero advierte: "procederemos del modo más seguro si construimos a priori cada species animalium y demostramos luego de qué modo se desvía, en cada una de estas especies, el respectivo proceso vital" (Entwurf einer allgemeinen Pathologie, II, Halle, p. 8); por su parte, Kieser pretende fundamentar su Sistema de la medicina en una filosofía entendida como "ley fundamental de la vida en general, así como de sus manifestaciones -también la enfermedad- persiguiendo con rigor inalterable hasta lo particular las manifestaciones morbosas individuales dadas por la experiencia" (System der Medicin zum Gebrauche bei akademischen Vorlesung und fiir practische Ärzte, I, Halle, p. IV.). Para más información puede consultarse el capítulo XIII de ARQUIOLA, E.; MONTIEL, L. (1993), pp. 335-348.

20) STARK, K.W. (1838). Allgemeine Pathologie oder allgemeine Naturlehre der Krankheit, II, Leipzig, p. 1365. 


\section{LUIS MONTIEL}

procesos vitales diferentes ${ }^{21}$. Sin duda es esto lo que hace que se oponga a un sistema nosológico como el del respetado Schönlein, que, como Bleker ha mostrado, es y quiere ser explícitamente clínico, aun cuando su profesión de fe empírica no agote la teoría de dicho sistema ${ }^{22}$. En todo caso, ese forzado empirismo le hace - piensa Stark - inhábil para conocer la auténtica entidad de la enfermedad, su fisiología ${ }^{23}$, es decir, para cumplir la consigna de aquellos que, aún teniendo que ser superados, han señalado un camino aceptable a la medicina alemana. En efecto: medicina científica - parece decir tácitamente Stark - equivale a medicina fisiológica; pero, si bien ese postulado margina los sistemas empíricos, tampoco la nosotaxia filosoficonatural sale bien parada del análisis que este autor hace de los sistemas precedentes. La clasificación "filosoficonatural" (no menciona autor alguno en concreto), basada en los tres sistemas responsables de la reproducción, la irritabilidad y la excitabilidad es, asegura, "no fisiológica" - unphysiologisch - dado que esos sistemas no existen realmente en la naturaleza ${ }^{24}$. Por otra parte, su ontologismo le aparta de manera radical de la patología físiológica soñada por la medicina filosoficonatural, que, explícitamente - también en el caso de Kieser- define el proceso morboso - Krankheitsprozess - como una "desviación" del proceso vital - Lebensprozess - normal25.

A la vista de lo anterior, da la impresión de que Stark se mueve con dificultad sobre un margen muy inestable entre un empirismo malfamado en el ambiente científico de la época, pero hacia el que, como clínico, siente querencia, y una voluntad de teoría que, en el panorama que contempla, colisiona a menudo con los datos suministrados por los sentidos. A este respecto resulta esclarecedora la actitud, que conocemos en autores contemporáneos más célebres — pienso, en primer término, en Bichat- de desconfianza hacia los instrumentos que pueden interponerse entre los sentidos y la naturaleza; explícitamente menciona Stark el microscopio y el laboratorio ${ }^{26}$. Cuanto más directa y totalizadora sea la observación, tanto más fiable será para un espíritu filosófico; esto es lo que, con su rechazo a las citadas técnicas, parece querer decir nuestro autor. Hay que revalidar la observación, la experiencia, pero no a cualquier precio. Pero, por otra parte, tampoco la teoría puede ser elevada al más alto rango, pues Kant nos ha enseñado que también el entendimiento tiene cierto carácter instrumental, que hace que yo vea las cosas "en mí", y no "en sí"; de manera

\footnotetext{
21 Ibid.

22 Schönlein se esfuerza en discernir los "síntomas esenciales" de los simplemente frecuentes, para lo cual intenta dar una base morfofuncional a su sistema, basando su clasificación en los "tejidos fundamentales del cuerpo" (BLEKER, J. (1981), pp. 138-139).

2.3 Ibid., pp. 1368-1369.

24 Ibid., p. 1368.

25 Cfr. ARQuiola, E.; Montiel, L. (1993), cap. XIII, pp. 335-348.

26 Ibid., 1367.
} 
que, en nombre de una deseable objetividad, Stark se ve obligado a rechazar como unphysiologisch la construcción intelectual que representa la patología filosoficonatural. En síntesis, lo que, para una filosofía que desee ser crítica, ya no resulta aceptable es un conocimiento mediado por instrumentos -incluído el entendimiento mismo con sus categorías- que pueden modificar la percepción de la realidad.

Lo repetiré: el lector de este capítulo, central desde el punto de vista conceptual, de la Allgemeine Pathologie, no puede dejar de experimentar la sensación de que su autor pelea con un problema realmente incómodo, al intentar conciliar observación y teoría, y que es precisamente ese empeño lo que le lleva a preferir las clasificaciones a cualquier otro método para abordar el estudio racional de la enfermedad y de las enfermedades. Como afirma al final de su ajuste de cuentas con los sistemas precedentes y contemporáneos, un sistema natural de las enfermedades que realmente merezca ese nombre debería permitir identificar "esencialmente" cada forma morbosa y, al propio tiempo, dar razón de la unidad englobante de todas ellas, lo que él llama, llevando al extremo su voluntad taxonómica, "el reino de las enfermedades"27. No puede resultar extraño que su esfuerzo resulte baldío.

\section{F. JAHN Y EL "REINO DE LAS ENFERMEDADES"}

Lo primero que hay que decir acerca de F. Jahn es que ha dejado muy claro el linaje al que asegura pertenecer. En su vasto estudio historicomédico sobre Sydenham ${ }^{28}$, considera al llamado "Hipócrates inglés" como profeta de la escuela historiconatural, y a Schönlein y Stark como fundadores de la misma ${ }^{29}$. Como el lector puede ver, este último dato es testimonio de la confusión que, ya en esa época, rodea al problemático grupo, pues, como queda dicho, Stark juzga crróneo el sistema de Schönlein. Por otra parte, Jahn parece no querer romper con la medicina filosoficonatural inmediatamente precedente. En este sentido cabe interpretar el motto con que arranca una de sus obras, fechada nada menos que en 1842, y que no es otro que una cita de Schelling que, eso sí, hace referencia a la necesidad de conocer los géneros morbosos ${ }^{30}$.

En relación con este asunto es Schönlein, en todo caso, quien se acerca más a la realidad en el sentir de Jahn: su sistema, dice, es entre los llamados "naturales" el

\footnotetext{
27 Ibid., pp. 1370-1371.

28 JAHN, F. (1840) Sydenham. Ein Beitrag zur wissenschaftlichen Medicin. Eisenach.

29) 29. Ibid., pp. V-VII

30) JAHN, F. (1842), Die abnormen Zustände des menschlichen Lebens als Nachbildungen und Wiederholungen normaler Zustände des Thierlebens. Eisenach und Wien.
} 


\section{LUIS MONTIEL}

más natural ${ }^{31}$. Este juicio favorable está en la base de su justificación de la prioridad epistemológica de la sistemática; prioridad que, como veremos, está matizada por el hecho de que la condición "natural" del sistema de las enfermedades que Jahn se plantea depende de su carácter "fisiológico"32. En esto Jahn coincide plenamente con Schönlein -recordemos su conocida clasificación de las enfermedades en "Morphen", "Haematosen" y "Neurosen"-, aun cuando discrepa de él al postular una clasificación dicotómica en lugar de la tripartita de Schönlein. Se basa, para ello, en una orientación más extremadamente fisiológica, dinámica, que la de quien llama su maestro, pues diluye las enfermedades clasificadas según un criterio anatomopatológico - las "morfonosis"33 - en el seno de dos grandes grupos, correspondientes a los dos grandes sistemas corporales, el vegetativo y el sensitivo. En esta bipartición se reencuentra, en todo caso, con su maestro, pues atribuye a la sangre el papel rector en el sistema vegetativo, e identifica el sensitivo con el sistema nervioso en su conjunto $^{34}$. Pero esta división le conduce, precisamente, al territorio más peligroso de los transitados por la Naturphilosophie, aquél en el que reina la ley de la polaridad y que, con tan gran facilidad, sirve de base a especulaciones de carácter axiológico. Jahn no es capaz de evitar la recaída en el concepto de "egoísmo", que ya estaba presente en Kieser, atribuyendo esta condición al sistema sensitivo en tanto que soporte de lo que en la vida de un ser es más individual, por oposición al "altruísmo" del sistema vegetativo, cuya función es el mantenimiento de lo específico ${ }^{35}$. No es,

31 JAHN, F. (1828). Ahnungen einer allgemeinen Naturgeschichte der Krankheiten. Eisenach, pp. 228-229.

32 JAHN, F. (1835). System der Physiatrik oder der hippokratischen Medicin, I. Eisenach, p. 49.

33 Adopto, al menos provisionalmente, para la traducción, los términos corrientes en la literatura en castellano -es decir, los usados por Laín y López Piñero. Este último cita, por la edición de Zurich de la Patología general de Schönlein — redactada por alumnos desconocidos- los términos "Morphonosen", "Hämatosen" y "Neuronosen". Bleker, en Op. cit., p. 66, escribe "Morphen", "Hämatosen" у "Neurosen". En la edición de St. Gallen - la que yo he podido manejar- se utilizan los vocablos "Morphen", "Hämatosen" y "Neuroflogosen". Cfr. Dr. J. L. Schönlein's Allgemeine und specielle Pathologie und Therapie, nach dessen Vorlesungen niedergeschrieben und herausgegeben von einigen seiner Zuhörer, Teil I (1841). St. Gallen, in Literatur-Comptoir.

34 En este punto puede considerarse a Jahn "retrógrado", por comparación con algunos Naturphilosophen cuyas obras he tenido ocasión de estudiar, como son Ph.F. von Walther y G.H. von Schubert, quienes adoptan tempranamente la distinción bichatiana de dos divisiones diferentes en el sistema nervioso, "sensitiva" una y "vegetativa" la otra. Cfr. MonTIEL, L. "Más allá del 'nacimiento de la clínica'. La comprensión de la Anatomía general de Bichat desde la Naturphilosophie". Actas del Symposion Internacional "1974: Inicios del idealismo alemán". (En prensa); MonTIEL, L. "Creativité de la pensée médicale: l'exemple des théories neurobiologiques du rêve dans la médecine romantique allemande". En: Debru, C. (Ed.) Les Sciences Physiologiques entre Biologie et Médecine. (En prensa).

35 JAHN, F. (1835), pp. 92-93. 
pues, extraño que Laín sitúe a Jahn, pese a la distancia cronológica, entre los Naturphilosophen ${ }^{36}$.

Sin embargo, del mismo modo que Bauer podía señalar, en el caso de Stark, una influencia científicamente "respetable" —la del método comparativo en el estudio de la anatomía ${ }^{37}$-, también en el de Jahn puede detectarse el influjo de una metodología prestigiosa en otro campo, a saber, el de las ciencias biológicas propiamente dichas, la botánica y la zoología. Los escritos de autores ya clásicos en este campo Linneo, Alexander von Humboldt- parecen estar detrás de algunos de los asertos del médico de Meiningen, por ejemplo el referente a la mayor densidad y variedad de formas de vida cuanto mayor sea la proximidad al nivel del mar, y el relativo al desplazamiento de algunas de estas especies desde los países cálidos hacia otros más fríos, al norte, ejemplos estos que hace extensivos, desde su perspectiva, a las enfermedades $^{38}$, pues como afirma axiomáticamente, "el mundo de la enfermedad Krankheitswelt - se comporta igual que los dos grandes reinos de la naturaleza"39. Cuando menos cabría atribuír a Jahn el mérito de sostener una concepción explícitamente ecológica de la salud y la enfermedad ${ }^{40}$, por más que esto le aleje del ideal de una fisiología del proceso vital alterado como la postulada por sus predecesores.

Y sin embargo, tal como yo mismo he subrayado en otro lugar, existe una continuidad entre el postulado fundamental de la Patología General que la Naturphilosophie pretende construir y la obra de F. Jahn: la medicina científica tiene que ser fisiológica; la patología, "fisiología de la enfermedad". Pero ingenuamente, Jahn parece haber pensado, como Stark, que la forma de hacer "fisiología de la enfermedad" consiste en reconocer a la enfermedad su propio Lebensprocess - proceso vital-, sustantivándola del modo que conocemos a través de la Parasitenlehre ${ }^{41}$. Lamentable error, que no le permitirá pasar siquiera, para la historiografía por venir, por precursor de una "mentalidad etiopatológica" como la profesada por Klebs, pues no es

36 LAIN, P. (1962). Historia de la medicina moderna y contemporánea. Barcelona, p. 440.

37 V. nota 18

38 JAHN, F. (1828), pp. 183-186

39 Ibid., p. 218.

40) Esta concepción, señalada por Tsouyopoulos para la medicina filosoficonatural en su conjunto, alcanza, en todo caso, una dimensión más concreta en el caso de Jahn. La autora alemana utiliza ese término para referirse a la concepción, cuyo origen rastrea en la obra de John Brown, de la salud y la enfermedad como estados, en cierto sentido "reactivos", condicionados por la acción del entorno. Cfr. Tsouyopoulos, N. (1982). Andreas Röschlaub und die Romantische Medizin. Die philosophischen Grundlagen der modernen Medizin. Stuttgart-New York, Gustav Fischer Verlag, especialmente pp. 200205.

41 Montiel, L. (1988). "El ontologismo nosológico de Ferdinand Jahn en la historia de la Patología". Actas del VIII Congreso Nacional de Historia de la Medicina, vol. III. Murcia, pp. 1448-1459. (p. 1453). 
"lucha por la existencia", sino "copia y repetición de formas de vida animales"42 lo que a la postre, constituye la enfermedad así entendida ${ }^{43}$.

Pero volvamos por un momento atrás, pues corremos el riesgo de pasar por alto un detalle extremadamente significativo que, además, puede ser rectamente valorado a la luz de lo anterior. Hemos visto que el mérito que Jahn encuentra en el sistema de Schönlein es su fundamento fisiológico, mientras que Stark lo censura justamente por lo contrario, es decir, por excesivamente empírico. Ante esta contradicción habría que preguntarse si Stark y Jahn hablan de la misma persona - y del mismo sistema médico-; o tal vez la pregunta debería ser otra, a saber, con qué mirada contempla cada uno la obra del presunto común maestro. Ello puede permitirnos aventurar una respuesta, que probablemente no será definitiva ni absolutamente satisfactoria, a la pregunta sobre esta fugaz resurrección de las nosologías en la Alemania del período Biedermeier.

\section{UN REINO QUE NO ES DE ESTE MUNDO.}

En esta diferente interpretación del pensamiento de Schönlein se ponen, a mi entender, de manifiesto las vacilaciones propias de este momento histórico. Los médicos de esta generación tienen que optar por un criterio de cientificidad y, en las figuras de Stark y Jahn, presuntamente alineados en la rama con mayor coherencia interna de la naturhistorische Schule - la parasitista- esta tensión se observa con extrema claridad. El primero de ellos, pese a su crítica al empirismo y su declarada voluntad "fisiológica", se decanta por una medicina basada en la observación que comparte algunos rasgos con el sensualismo profesado por Bichat, en tanto que Jahn, que se autotitula discípulo suyo y de Schönlein, manifiesta una fuerte querencia por una orientación fisiológica de raigambre filosoficonatural. Por esta razón es forzoso coincidir con la principal tesis de Bleker, a saber, que, en general, los médicos de la "escuela historiconatural" no propiciaron el paso de la Naturphilosophie a la $\mathrm{Na}$ turwissenschaft entendida como ciencia hecha en el laboratorio, sino a una propuesta diferente de medicina clínica, para la que las ciencias naturales no constituirían sino una propedéutica o un medio auxiliar ${ }^{44}$. En todo caso, la aceptación de esta tesis

42 Cfr. el título - y, desde luego, el contenido- de su obra de 1842.

4. Es necesario señalar que W. Pagel le atribuye el papel de precursor de la patología celular de Virchow. Aunque detallado y riguroso, el trabajo en que defiende esta tesis es susceptible de discusión, al menos en el nivel de la interpretación de los datos. Por otra parte, con los mismos argumentos que Pagel esgrime en favor de esa asociación, podrían defenderse otras, remontándonos a la patología filosoficonatural, especialmente a la de Kieser. Cfr. PAGEL, W. (1945). "The speculative basis of modern Pathology. Jahn, Virchow and the philosophy of Pathology". Bulletin of the History of Medicine, XVIII-I, pp. 1-43.

44 Bleker, J. (1981), pp. 133-134. 
general no debe producirse sin alguna matización, en los casos concretos estudiados. Pienso que la voluntad fisiológica de estos dos autores es muy fuerte, aunque secundaria, en su escala de valores, respecto de la observación, descripción y ordenación de las entidades morbosas y que, por otra parte, se manifiesta, como queda dicho, con intensidad diferente en cada uno de ellos; así, Stark sería, o se tendría por más "nosógrafo", y Jahn por más "fisiopatólogo", aunque en un sentido aún muy próximo al de la Naturphilosophie. Insisto en señalar que esto refleja, más que la realidad, la voluntad de cada uno de los autores. En esta perspectiva, aunque los dos dicen utilizar un método historiconatural, lo cierto es que ninguno de ellos lo hace plenamente, pues la diferente remisión de ambos a la fisiología hace que se instalen en la órbita de la recién nacida Biología, conceptualmente diferente de la clásica Historia Natural. Y, para esta nueva ciencia, las clasificaciones ocupan un lugar epistemológicamente secundario y radicalmente distinto, tanto en su orientación como en el valor que se le concede, al que poseían en la Historia Natural anterior a Lamarck y Treviranus $^{45}$. Así pues, aunque innovadores en cuanto a la posición en que se sitúan, Stark y Jahn no consiguen serlo plenamente en cuanto a los objetivos y al método, pues aspiran todavía a algo a lo que, deliberadamente, renunciarán los autores que, efectivamente, llevarán la medicina al campo de las Naturwissenschaften: la construcción de un sistema; más específicamente, de un "sistema de las enfermedades". Serán otros autores, capaces de interpretar más rectamente algunas consignas de la Naturphilosophie, quienes elijan el camino acertado: el análisis de los procesos dinámicos en que la salud y la enfermedad consisten. Estos investigadores se fijarán una sucesión de objetivos parciales más modestos, abandonando lo que constituyó el, a mi juicio, rasgo fundamental de al menos dos de los miembros de la compleja "escuela historiconatural": perseguir un reino, el de las enfermedades, que no es de este mundo.

45 Existe abundante bibliografía a este respecto. El lector pueden encontrar una buena parte de ella, así como un análisis del tránsito de la Historia Natural a la Biología en Francia y Alemania, en el cap. VII de: ARQUiOLA, E. ; MONTIEL, L. (1993), pp. 155-167. 